\title{
Características epidemiológicas y clínicas de las infecciones por dengue y zika durante el fenómeno de El Niño Costero de 2017 en Chincha, Perú
}

\author{
Epidemiological and clinical characteristics of dengue and zika infections during the \\ EI Niño Costero phenomenon in 2017 in Chincha, Peru
}

Alexander Reátegui ${ }^{1}$, Néstor Falcón ${ }^{1,3}$

\section{Resumen}

\begin{abstract}
El objetivo del estudio fue determinar las características clínicas y epidemiológicas de los casos confirmados de zika y dengue, presentados durante el Fenómeno de El Niño Costero, periodo enero-junio de 2017 y que fueran atendidos en el Centro de Salud del distrito Pueblo Nuevo, provincia de Chincha, Ica, Perú. Se diseñó un estudio observacional, descriptivo y retrospectivo que sistematizó información demográfica, signos clínicos y tiempo transcurrido entre el inicio de estos y la investigación, notificación y confirmación de los casos. Se recuperaron 130 casos confirmados positivos a zika y 44 a dengue. La mayor cantidad de casos se reportaron entre las semanas epidemiológicas 12 y 15 y en mujeres ( $76.9 \%$ de zika y $63.6 \%$ de dengue). La mayor cantidad de casos de zika y dengue se presentó en el grupo etario de 20 -29 años (30.0 y 27.3\%, respectivamente). Los signos clínicos más frecuentes en ambos casos fueron exantema, cefalea, mialgias, dolor ocular/retrocular y artralgia/poliartralgia. La relación entre el comportamiento temporal de los casos y las intervenciones realizadas muestran que la aplicación de la nebulización tuvo un mayor efecto, disminuyendo la cantidad de casos confirmados después de la aplicación. Se concluye que un reconocimiento y notificación temprana de la presencia de dengue y zika, así como el uso de las nebulizaciones para control del vector, tienen un impacto directo sobre la cantidad de nuevos casos de estas enfermedades en una zona no endémica.
\end{abstract}

Palabras clave: cambo climático, metaxénicas, salud pública, zoonosis

${ }^{1}$ Laboratorio de Epidemiología y Salud Pública en Veterinaria, Facultad de Medicina Veterinaria y Zootecnia, Universidad Peruana Cayetano Heredia, Lima, Perú

${ }^{2}$ E-mail: nestor.falcon@upch.pe

Recibido: 7 de abril de 2020

Aceptado para publicación: 30 de enero de 2021

Publicado: 24 de abril de 2021 
The aim of this study was to determine the clinical and epidemiological characteristics of the confirmed cases of Zika and dengue, presented during the El Niño Costero phenomenon, period January-June 2017 and that were treated at the Health Centre of the Pueblo Nuevo district, province of Chincha, in Ica, Peru. An observational, descriptive and retrospective study was designed that systematized demographic information, clinical signs and the time elapsed between the beginning of these and the investigation, notification and confirmation of the cases. In total, 130 confirmed positive cases for Zika and 44 for dengue were recovered. The greatest number of cases were reported between epidemiological weeks 12 and 15 and in women (76.9\% of Zika and $63.6 \%$ of dengue). The greatest number of Zika and dengue cases occurred in the age group of 20-29 years (30.0 and $27 \%$, respectively). The most frequent clinical signs in both cases were exanthema, headache, myalgia, ocular/retrocular pain and arthralgia/polyarthralgia. The relationship between the temporal behaviour of the cases and the interventions carried out show that the nebulization application had a greater effect, reducing the number of confirmed cases after the application. It is concluded that early recognition and notification of the presence of dengue and zika, as well as the use of nebulization for vector control have a direct impact on the number of new cases of these diseases in a non-endemic area.

Key words: climate change, metaxenics, public health, zoonosis

\section{INTRODUCCIÓN}

Las enfermedades producidas por el virus del dengue (DENV) y zika (ZIKV) se encuentran agrupadas dentro de las denominadas metaxénicas, perteneciendo al grupo de los arbovirus, género Flavivirus y familia Flaviviridae. Estas son transmitidas por la picadura del mosquito Aedes aegypti y trasmitido de un huésped humano infectado a uno susceptible (Bogantes y Montoya, 2016).

El virus del Dengue contiene cuatro serotipos serológicamente diferentes DEN1, DEN-2, DEN-3 y DEN-4, siendo los serotipos DEN-2 y DEN3 los reportados en la mayoría de casos graves y con fallecimiento de los afectados (Lindenbach et al., 2007). Los signos clínicos se manifiestan de acuerdo con el serotipo infeccioso, respuesta inmune, tipo racial, etc. (Cáceres, 2003). El periodo de incubación del dengue fluctúa en- tre 4 a 10 días y los signos clínicos persisten entre 2 a 7 días. Entre los síntomas más frecuentes se encuentra la fiebre $\left(40^{\circ} \mathrm{C}\right)$, cefalea, náuseas, vómitos, dolor en músculos y articulaciones, ganglios linfáticos reactivos y salpullido (Martínez, 2008). Los pacientes infectados con el serotipo DEN-2 presentan, además, hematemesis, hemorragias en encías, dolor abdominal intenso y vómitos frecuentes, por lo que la atención medica es indispensable para reducir el riesgo de muerte (Cabezas, 2005; Malagon et al., 2011).

En el caso de zika se han identificado diferentes serotipos que se han agrupado por regiones: los africanos (4 serotipos) y los asiáticos (3 serotipos) (Ochoa-Ortega, 2015; Shapshak et al., 2015). El virus del Zika es una enfermedad emergente en América, siendo diagnosticada por primera vez en Brasil en 2015, reportándose meses después en transmisiones autóctonas en Colombia (Enfissi et al., 2016). El ZIKV no solo se 
transmite por la picadura del mosquito Aedes. La transmisión transplacentaria es frecuente, pudiendo causar microcefalia y malformaciones del SNC en el feto, así como originar abortos espontáneos (Aspilcueta et al., 2017). También se presenta la transmisión sexual, debido a que los espermatozoides del hombre infectado poseen una alta carga viral replicativa y ARN viral (Petersen et al., 2016). La sintomatología es similar a la causada por el virus del dengue y chikungunya, tiene un periodo de incubación de 2 a 7 días, con manifestaciones clínicas de fiebre, exantema maculopapuloso, conjuntivitis no supurada, mialgias y/o artralgias, que persisten entre 2 a 7 días (Cabrera et al., 2017).

A inicio de 2017 se presentaron intensas lluvias en varias regiones del Perú, afectando principalmente los departamentos costeros, evento climatológico que se denominó Fenómeno de El Niño Costero. Entre enero y junio de ese año, en el distrito de Pueblo Nuevo en Chincha (Ica, Perú) se enfrentó a un brote de dengue y zika concomitante con este fenómeno, lo que favoreció la presencia de un escenario epidemiológico III, que hizo necesaria la intervención urgente para evitar la diseminación de los casos clínicos. En ese contexto, el objetivo del estudio fue determinar las características epidemiológicas y clínicas de los casos confirmados de zika y dengue que fueron atendidos en el Centro de Salud del distrito Pueblo Nuevo, durante el Fenómeno de El Niño Costero de 2017.

\section{Materiales y MéTodos}

El estudio se realizó en el Centro de Salud del distrito de Pueblo Nuevo, provincia de Chincha, departamento de Ica, Perú. El análisis de datos se realizó en el Laboratorio de Epidemiología y Salud Pública en Veterinaria de la Facultad de Medicina Veterinaria y Zootecnia de la Universidad Peruana Cayetano Heredia (UPCH), en Lima. La investigación correspondió a un estudio de tipo observacional, descriptivo y retrospectivo.
La población objetivo estuvo conformada por las historias clínicas de pacientes con diagnóstico definitivo a la prueba de aislamiento viral para las enfermedades de dengue o zika (MINSA, 2017); independientemente del género y edad, domiciliados en la zona de estudio durante el Fenómeno de El Niño Costero y que fueron atendidos en el Centro de Salud de Pueblo Nuevo durante el periodo de la semana epidemiológica 8 a la semana 36 de 2017. Se desarrolló una ficha de registro de información considerando las variables epidemiológicas y manifestaciones clínicas de los casos confirmados de dengue y zika.

La información fue analizada mediante el paquete estadístico STATA v. 13.0. Los resultados fueron resumidos utilizando estadígrafos descriptivos (frecuencia absoluta y relativa). La asociación entre los datos demográficos de grupo etario $(<10,10-14,15-$ $19,20-29,30-39,40-49,50-59 \mathrm{y} \geq 60$ años) y género (masculino y femenino), y género (masculino y femenino), con el diagnostico confirmado se realizó mediante la prueba de Chi Cuadrado. Los resultados se presentan en cuadros comparativos de frecuencias. El estudio fue aprobado por el Comité Institucional de Ética de la UPCH con constancia N. ${ }^{\circ} 038-04-18$.

\section{Resultados}

Se obtuvieron 130 casos confirmados de zika y 44 de dengue. La distribución de los casos según semana epidemiológica muestra que la mayor cantidad de casos se presentaron, para ambas enfermedades, entre las semanas 12 y 15. Se observó que el índice aédico (porcentaje de casas positivas al Aedes aegypti en la localidad) y los casos de zika estaban en aumento aun cuando se desarrollaban las actividades de abatización del mosquito (semana 6 a la 19). En cambio, ambas variables disminuyen cuando se realizó la campaña de nebulización, la que se realizó entre la semana epidemiológica 15 a la 


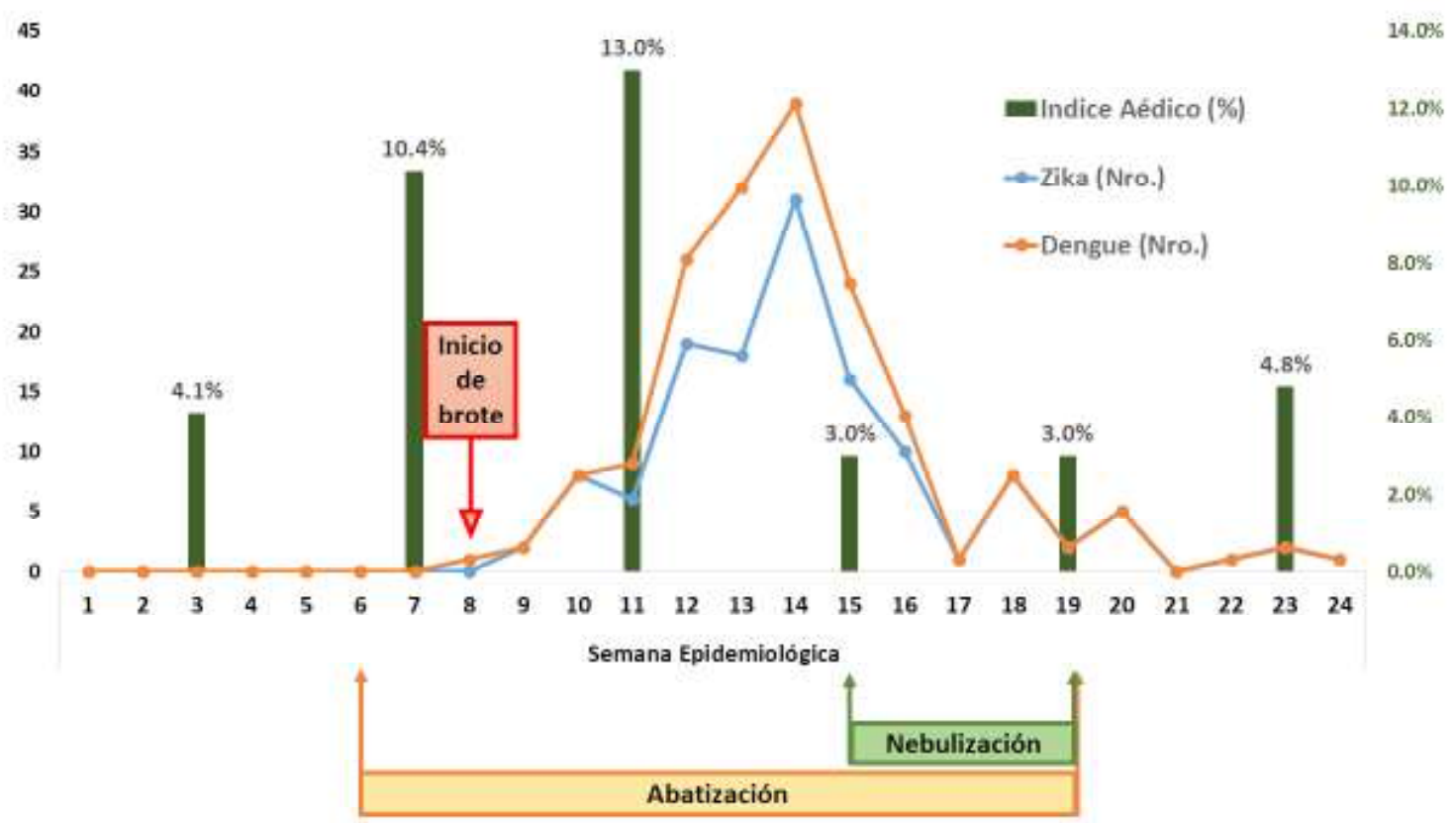

Figura 1. Relación entre medidas de intervención, índice aédico (\%) y distribución temporal $\left(\mathrm{N}^{\circ}\right)$ de los casos de zika y dengue durante el Fenómeno de El Niño Costero. Centro de Salud del distrito de Pueblo Nuevo - Chincha, 2017

19 (entre el 4 de abril hasta el 9 de mayo). La distribución de la enfermedad y el efecto de las intervenciones se presentan en la Figura 1.

La mayor cantidad de casos se reportaron en personas del sexo femenino tanto para zika $(76.9 \%)$ como para dengue (63.6\%), no observándose diferencia significativa en la proporción de afectados por sexo y tipo de enfermedad. Entre los casos de dengue en mujeres $(n=28)$, una se encontraba en el puerperio, mientras que para los casos mujeres con zika $(n=100), 25$ estaban gestantes y 9 en puerperio.

El grupo etario con la mayor cantidad de casos fue el de 20-29 años para el caso de zika $(30.0 \%)$ y para dengue $(27.3 \%)$ (Cuadro 1). La edad promedio de los afectados por zika fue de 28.0 años (desviación estándar de 17.3 años) y los afectados por dengue fue de 38.6 años (desviación estándar de 15.0 años). No se encontró asociación entre la distribución de los grupos etarios y la enfermedad estudiada.

Los signos clínicos más frecuentes en las dos enfermedades fueron exantema, cefalea, mialgias, dolor ocular/retrocular y artralgia/poliartralgia. Se halló asociación entre la presentación de exantema con el tipo de enfermedad en estudio (Cuadro 2).

\section{Discusión}

Los 174 diagnósticos de enfermedades metaxénicas (zika y dengue) evaluados durante el Fenómeno de El Niño Costero en la zona en estudio solo corresponden a las mues- 
Cuadro 1. Distribución de los casos de zika y dengue por grupo etario y sexo durante el Fenómeno de El Niño Costero (Centro de Salud de Pueblo Nuevo, Chincha - 2017)

\begin{tabular}{|c|c|c|c|c|}
\hline \multirow{2}{*}{ Variable } & \multicolumn{2}{|c|}{ Zika } & \multicolumn{2}{|c|}{ Dengue } \\
\hline & $\mathrm{n}$ & $\%$ & $\mathrm{n}$ & $\%$ \\
\hline \multicolumn{5}{|l|}{ Sexo } \\
\hline Femenino & 100 & 76.9 & 28 & 63.6 \\
\hline Masculino & 30 & 23.1 & 16 & 36.4 \\
\hline \multicolumn{5}{|l|}{ Grupo etario (años) } \\
\hline$<10$ & 16 & 12.3 & 1 & 2.3 \\
\hline $10-14$ & 9 & 6.9 & - & - \\
\hline $15-19$ & 16 & 12.3 & 2 & 4.5 \\
\hline 20- 29 & 39 & 30.0 & 12 & 27.3 \\
\hline 30- 39 & 22 & 17.0 & 8 & 18.2 \\
\hline 40- 49 & 13 & 10.0 & 11 & 25 \\
\hline $50-59$ & 6 & 4.6 & 5 & 11.4 \\
\hline$\geq 60$ & 9 & 6.9 & 5 & 11.4 \\
\hline
\end{tabular}

Cuadro 2. Frecuencia de signos clínicos reportados en casos de zika y dengue durante el Fenómeno de El Niño Costero (Centro de Salud de Pueblo Nuevo, Chincha - 2017)

\begin{tabular}{lccccc}
\hline \multirow{2}{*}{ Signos clínicos } & \multicolumn{2}{c}{ Zika } & \multicolumn{2}{c}{$\begin{array}{c}\text { Dengue } \\
(\mathrm{n}=44)\end{array}$} & \multirow{2}{*}{ Valor $\mathrm{p}$} \\
\cline { 2 - 5 } & $\mathrm{n}$ & $\%$ & $\mathrm{n}$ & $\%$ & \\
\hline Exantema & 119 & 91.5 & 44 & 100.0 & 0.0452 \\
Cefalea & 115 & 88.5 & 43 & 97.7 & 0.0692 \\
Mialgias & 92 & 70.8 & 35 & 79.5 & 0.2548 \\
Dolor ocular o retro ocular & 59 & 45.4 & 22 & 50.0 & 0.6000 \\
Artralgias/poliartralgia & 55 & 42.3 & 23 & 52.3 & 0.2471 \\
Fiebre & 33 & 25.4 & 5 & 11.4 & 0.0521 \\
Conjuntivitis (ojos rojos) & 24 & 18.5 & 8 & 18.2 & 0.9641 \\
Erupción cutánea & 5 & 3.9 &.-- &.-- & 0.1785 \\
Diarrea & 3 & 2.3 &.-- &.-- & 0.2919 \\
Nauseas & 3 & 2.3 & 1 & 2.3 & 1.0000 \\
Artritis & 2 & 1.5 &.-- &.-- & 0.4115 \\
Dolor lumbar & 1 & 0.8 &.-- &.-- & 0.5113 \\
\hline
\end{tabular}


tras que contaban con diagnóstico confirmado por el Instituto Nacional de Salud, por lo que el estudio no necesariamente refleja la magnitud de las enfermedades que se presentaron en el lugar de estudio. Además de las condiciones climatológicas producto del Fenómeno de El Niño Costero que habrían favorecido la reproducción de $A$. aegypti, vector del ZIKV y DENV, se encontraban las condiciones socioeconómicas de la zona, la cual sufrió un terremoto en 2007, que habría impedido la reposición completa de los servicios de agua y desagüe.

En ambas enfermedades, la mayor cantidad de casos se presentaron en las semanas epidemiológicas correspondientes al mes de marzo. Considerando que el periodo de incubación del dengue se encuentra entre 7 a 14 días (Barón y Munguía, 2017; Cabezas et al., 2015) y el de zika entre 3 a 12 días (Maguiña y Galan-Rodas, 2016), la mayor exposición humana al vector se habría producido en la última semana de enero y las primeras semanas de febrero. Ello estaría asociado a que en esas épocas se alcanzaron las temperaturas más altas del verano y una mayor precipitación pluvial, que favoreció la reproducción del vector y consecuentemente el del índice aédico. Estas condiciones habrían favorecido el aumento del índice de picaduras y el aumento de la probabilidad de transmisión de los virus desde un hospedero enfermo a uno sano susceptible.

Bisset et al. (2007) mencionan que debido a que no existe una solución segura para el control del dengue (lo que se puede hacer extensivo a las demás enfermedades metaxénicas transmitidas por $A$. aegypti), es indispensable controlar la reproducción del vector artrópodo para prevenir o reducir la transmisión. Las medidas de control aplicadas en este caso tuvieron diferentes respuestas. La abatización se realizó entre la semana 6 y la 19 para prevenir la proliferación de A. aegypti; sin embargo, a juzgar por los resultados, esta actividad no habría tenido los efectos esperados debido a que dos semanas más tarde se iniciaron los brotes de dengue y zika. El realizarlo de manera no sostenida, con participación parcial de la población (solo incluyó 1913 viviendas) y la no eliminación de los reservorios de huevos fuera de los hogares, habrían contribuido a que esta medida de control no sea eficiente.

La nebulización, por otra parte, se aplicó entre la semana epidemiológica 15 a la 19 (4 de abril - 9 de mayo) en 117908 viviendas a nivel de Chincha en general, considerando tres repeticiones con intervalos de 5 días y haciendo uso de camiones nebulizadores, además de 68 nebulizaciones manuales complementarias. Esta intervención produjo una disminución del índice aédico y, por lo tanto, los casos de dengue y zika.

Las nebulizaciones son utilizadas cuando las medidas preventivas como el abate y la eliminación de los reservorios de mosquitos resultan insuficientes. Bisset et al. (2007) mencionan que los insecticidas contra mosquitos adultos solo tienen justificación en caso de epidemia o para interrumpir la transmisión cuando existen altos niveles de infestación, en donde se suele usar principalmente los piretroides. Amparado en la situación epidemiológica que se presentaba en la zona de estudio se justificó el uso de las nebulizaciones, debido a que las medidas de prevención no pudieron contener la aparición del brote de zika y dengue.

La mayor cantidad de casos se reportaron en mujeres, tanto para zika (76.9\%) como para dengue (63.6\%). Aunque el modelo de estudio realizado a base de casos confirmados no permite determinar una asociación entre sexo y probabilidad de infección, los resultados de otras investigaciones en este sentido son diversos. Así, un estudio realizado por Aspilcueta et al. (2017) también encuentra una mayor cantidad de casos de zika en mujeres. En contraste, un estudio de factores de riesgos asociados a la infección por dengue en Anzoátegui, Venezuela, no encontró relación entre el sexo y la presentación de la enfermedad (Rivera et al., 2011). De la misma forma, la edad de los 
afectados fue amplia, de allí que tampoco se pudo determinar alguna relación. Sin embargo, Kantor (2016) menciona tener en cuenta que por la forma de transmisión del dengue y zika, sería más importante la exposición por prácticas antropogénicas que los factores demográficos de los individuos.

Los signos clínicos registrados en las historias clínicas no son suficientes para discriminar los casos de dengue y zika, por lo que se requiere la confirmación del laboratorio para determinar el tratamiento y pronóstico adecuado (Gubler, 1998; Lin et al., 2002; Oishi et al., 2007; Kyle y Harris, 2008). La importancia del diagnóstico del zika está asociada con el individuo afectado. El cuadro clínico es muy semejante al dengue no grave y al chikungunya (Rivera, 2014), pero en caso se produzca la infección en una embarazada puede conllevar al nacimiento de niños con microcefalia (Zanluca et al., 2015). También se encuentran asociados a la presentación del Síndrome de Guillain-Barré en 1 de 1000 casos sintomáticos (Mlakar, 2016),

Al no disponerse de una vacuna para proteger a la población humana y siendo la picadura del vector la principal forma de transmisión del dengue y zika, las estrategias preventivas deben estar dirigidas a disminuir la población del $A$. aegypti, evitando su reproducción.

\section{Conclusiones}

- La abatización del mosquito Aedes aegypti no representó una medida eficiente para el control de la reproducción del vector observándose un aumento del número de casos confirmados de zika y dengue durante las primeras semanas de aplicada dicha intervención.

- La nebulización fue la medida más eficiente para el control de la población del vector $A$. aegypti lo que se reflejó en una disminución del número de casos confirmados de zika y dengue.
- Los signos clínicos que se presentaron con mayor frecuencia en ambas enfermedades fueron exantema, cefalea, mialgias, dolor ocular/retrocular y artralgia/poliartralgia; pero se requiere la confirmación de laboratorio para determinar la enfermedad específica.

\section{Literatura Citada}

1. Aspilcueta D, Benites C, Calderón M, Calderón J. 2017. Infección por zika en el Perú: de amenaza a problema de salud. Rev Peru Ginecol Obstet 63: 57-64.

2. Barón A, Munguía I. 2017. Dengue, chikungunya, zika y mayaro, amenazas para la salud en Honduras. Rev Fac Cienc Med 14: 44-57.

3. Bisset JA, Rodríguez MM, Fernández D, Palomino M. 2007. Resistencia a insecticidas y mecanismos de resistencia en A. aegypti (Diptera: Culicidae) de 2 provincias del Perú. Rev Cubana Med Trop 59: 202-208.

4. Bogantes M, Montoya W. 2016. ¿Dengue, Chikungunya o Zika? Rev Clín Escuela Med Univ Costa Rica 6: 1-6.

5. Cabezas C. 2005. Dengue en el Perú: aportes para su diagnóstico y control. Rev Peru Med Exp Salud Pública 22: 212-228.

6. Cabezas C, Fiestas V, GarcíaMendoza M, Palomino M, Mamani E, Donaires F. 2015. Dengue en el Perú: a un cuarto de siglo de su reemergencia. Rev Peru Med Exp Salud Pública 32: 146-156.

7. Cabrera H, Vega J, Miranda, J, Martínez, S, García D, Martínez J. 2017. Infección por virus Zika como un reto para la Salud Pública. Rev Méd Electr 39: 304-312.

8. Cáceres O. 2003. Detección rápida de los serotipos del virus dengue en el mosquito Aedes aegypti. Rev Peru Med Exp Salud Pública 20: 156-158.

9. Enfissi A, Codrington J, Roosblad J, Kazanji M, Rousset D. 2016. Zika virus genome from the Americas. Lancet 
387: 227-228. doi: 10.1016/S01406736(16)-00003-9

10. Gubler D. 1998. Dengue and dengue hemorrhagic fever. Clin Microbiol Rev 11: 480-496.

11. Kantor IN. 2016. Dengue, Zika y chikungunya. Medicina (Buenos Aires) 76: 93-97.

12. Kyle J, Harris E. 2008. Global spread and persistence of dengue. Annu Rev Microbiol 62: 71-92. doi: 10.1146/ annurev.micro.62.081307.163005

13. Lin C, Lei H, Shiau A, Liu H, Yeh T, Chen $S$, et al. 2002. Endothelial cell apoptosis induced by antibodies against dengue virus nonstructural protein 1 via production of nitric oxide. J Immunol 169: 657-64. doi: 10.4049/jimmunol.169.2.657

14. Lindenbach B, Thiel H, Rice C. 2007. Flavivirus: the virus and their replication. In: Knipe D, Howley P (eds). Fields Virology. Philadelphia, USA: Lippincott Williams \& Wilkins. P 1101-1152.

15. Maguiña C, Galán-Rodas E. 2016. El virus Zika: una revisión de literatura. Acta Médica Peruana 33: 35-41.

16. Malagon N, Padilla J, Rojas D. 2011. Guía de atención clínica integral del paciente con dengue. Infection 15:293-301.

17. Martínez E. 2008. Dengue. Estudos avançados 22:33-52. doi: 10.1590/S010340142008000300004

18. [MINSA] Ministerio de Salud. 2017. Norma técnica de salud para la vigilancia epidemiológica y diagnóstico de laboratorio de dengue, chikungunya, zika y otras arbovirosis en el Perú. Lima: MINSA. 90p [Internet]. Disponible en: https://www.dge.gob.pe/portal/docs/ tools/arbovirosis18.pdf

19. Mlakar J, Korva M, Tul N, Popovice M, Poljšak-Prijatelj M, Mraz J, Kolenc $M$, et al. 2016. Zika virus associated- with microcephaly. New Engl J Med 374: 951-958. doi: 10.1056/NEJMoa1600651

20. Ochoa-Ortega M, Casanova-Moreno M, Díaz-Domínguez M. 2015. Análisis sobre el dengue, su agente transmisor y estrategias de prevención y control. Rev Arch Méd Camagüey 19: 189-202.

21. Oishi K, Saito M, Mapua C, Natividad F. 2007. Dengue illness: clinical features and pathogenesis. J Infect Chemother 13: 125-133. doi: 10.1007/ s10156-007-0516-9

22. Petersen LR, Jamieson DJ, Powers AM, Honein MA. 2016. Zika virus. New Engl J Med 374: 1552-1563. doi: 10.1056/NEJMra1602113

23. Rivera O. 2014. Aedes aegypti, virus dengue, chinkugunia, zika y el cambio climático. Máxima alerta médica y oficial. Redvet 15(10). [Internet]. Disponible en: https://www.redalyc.org/pdf/636/ 63637999001.pdf

24. Rivera A, Rodríguez A, Meléndrez, E. 2011. Factores de riesgos asociados a la infección por dengue en San Mateo, Anzoátegui, Venezuela. Rev Cubana Med Gen Integr 27: 388-395.

25. Shapshak P, Somboonwit C, Foley B, Alrabaa S, Wills T, Sinnott J. 2015. Zika virus. Global virology I - Identifying and investigating viral diseases. In: Shapshak P, Sinnott TJ, Charurut Somboonwit H, Kuhn J (eds). New York, USA: Springer $\mathrm{p} 477-500$.

26. Zanluca C, de Melo VCA, Mosimann $A L P$, dos Santos GIV, dos Santos CND, Luz K. 2015. First report of autochthonous transmission of Zika virus in Brazil. Mem I Oswaldo Cruz 110: 569572. doi: 10.1590/0074-02760150192 